Canad. Math. Bull. Vol. 21 (3), 1978

\title{
A REMARK ON COHERENT OVERRINGS
}

\author{
BY
}

IRA J. PAPICK

1. Introduction. Throughout this note, let $R$ be a (commutative integral) domain with quotient field $K$. A domain $S$ satisfying $R \subseteq S \subseteq K$ is called an overring of $R$, and by dimension of a ring we mean Krull dimension. Recall [1] that a commutative ring is said to be coherent if each finitely generated ideal is finitely presented.

In [2], as a corollary of a more general theorem, Davis showed that if each overring of a domain $R$ is Noetherian, then the dimension of $R$ is at most 1 . (This corollary is the converse of a version of the Krull-Akizuki Theorem [5, Theorem 93], and can also be proved directly by using the existence of valuation rings dominating finite chains of prime ideals [4, Corollary 16.6].) It is our purpose to prove that if $R$ is Noetherian and each overring of $R$ is coherent, then the dimension of $R$ is at most 1. We shall also indicate some related questions and examples.

Any unexplained terminology is standard as in [4] and [5].

2. THEOREM 1. If $R$ is Noetherian and each overring of $R$ is coherent, then the dimension of $R$ is at most 1 .

Proof. Assume the dimension of $R$ is greater than 1. By localizing at a height two prime and then passing to the integral closure, we may assume $R$ is a 2-dimensional integrally closed Noetherian domain [7,33.12]. Hence, by localizing again, we may assume $R$ is a local 2-dimensional Macaulay ring [5, Ex. 25, p. 104] with maximal ideal $M$. Let $x, y$ be an $R$-sequence of length two in $M$ and note that $y, x$ is also an $R$-sequence, since $R$ is a domain:

Let $J=\left\{x^{n} / y^{n-1}: n \geq 2, n\right.$ an integer $\}$ and consider the overring $S=R[J]$ of $R$. Observe that any element $f \in S$ can be written

$$
f=r_{0}+r_{1} x^{n_{1}} / y^{n_{1}-1}+\cdots+r_{t} x^{n_{t}} / y^{n_{t}-1},
$$

where $r_{0} \in R, \quad r_{i} \in R \backslash R y$ for $i \geq 1$ and $2 \leq n_{1}<\cdots<n_{t}$. (In simplifying an expression of the type $v_{0}+v_{1} x^{a_{1}} / y^{b_{1}}+\cdots+v_{s} x^{a_{s}} / y^{b_{s}}, v_{i} \in R, 1 \leq b_{i}<a_{i}$ for each $i$, we use the convention of first reducing the fractions appearing in that expression to the form $v x^{m} / y^{m-1}$, where $v \in R \backslash R y, m \geq 2$ or to elements of $R$; then we combine like terms and repeat the process if necessary until reaching an element of the form (1).) We claim that $S$ is not coherent. To substantiate

Received by the editor September 30, 1976. 
this claim, it is sufficient by [1, Theorem 2.2] to prove that $\left(x^{2} / y: x^{3} / y^{2}\right)=I$ is a non-finitely generated ideal of $S$. To help achieve this end we show that $I=(x, y) S+J S$. It is easily shown that the right hand side is contained in the left, so we focus on the reverse inclusion. Let $f \in I$. Then for some

$$
g=r_{0}+r_{1} x^{n_{1}} / y^{n_{1}-1}+\cdots+r_{t} x^{n_{t}} / y^{n_{t}-1}, \text { where } r_{0} \in R,
$$

$r_{i} \in R \backslash R y$ for $i \geq 1$, and $2 \leq n_{1}<\cdots<n_{t}$, we have

$$
f=g(y / x)=r_{0} y / x+(y / x)\left(r_{1} x^{n_{1}} / y^{n_{1}-1}+\cdots+r_{t} x^{n_{t}} / y^{n_{\mathrm{t}}-1}\right) .
$$

Hence, $r_{0} y / x \in S$; and so,

$$
r_{0}=v_{0} x / y+v_{1} x^{m_{1}} / y^{m_{1}-1}+\cdots+v_{s} x^{m_{s}} / y^{m_{s}-1},
$$

where $v_{0} \in R, v_{i} \in R \backslash R y$ for $i \geq 1$ and $3 \leq m_{1}<\cdots<m_{s}$. Multiplication of this equation by $y^{m_{s}-1}$ shows that $y^{m_{s}-1} r_{0} \in x R$, and thus $r_{0} \in x R$. Therefore $f \in$ $(x, y) S+J S$, and the desired equality is attained.

To complete the proof we show that $I$ is not finitely generated. If $I$ were finitely generated there would exist a finite subset $W=$ $\left\{x^{\alpha_{1}} / y^{\alpha_{1}-1}, \ldots, x^{\alpha_{w}} / y^{\alpha_{w}-1}\right\}$ of $J$ with $2 \leq \alpha_{1}<\cdots<\alpha_{w}$ so that $I=(x, y) S+W S$. Thus, for some $f_{i} \in S$,

$$
x^{\alpha_{w}+1} / y^{\alpha_{w}}=f_{1} x+f_{2} y+f_{3}\left(x^{\alpha_{1}} / y^{\alpha_{1}-1}\right)+\cdots+f_{w+2}\left(x^{\alpha_{w}} / y^{\alpha_{w}-1}\right) .
$$

By expanding and then simplifying in accordance to our convention, we have

$$
x^{\alpha_{w}+1} / y^{\alpha_{w}}=r_{0}+r_{1} x^{n_{1}} / y^{n_{1}-1}+\cdots+r_{t} x^{n_{t}} / y^{n_{t}-1},
$$

where $r_{0} \in R, r_{i} \in R \backslash R y$ for $i \geq 1$, and $2 \leq n_{1}<\cdots<n_{t}$.

CASE 1. $n_{t} \geq \alpha_{W}+1$. Under this assumption, it is not hard to see from equations (2) and (3) that $r_{t} \in R x$. Multiplication of equation (3) by $y^{n_{t}-1}$ shows that

$$
x^{\alpha_{w}+1}\left(y^{\left(n_{t}-1\right)-\alpha_{w}}-r_{t} x^{n_{t}-\left(\alpha_{w}+1\right)}\right) \in R y .
$$

But this implies that $y^{n_{t}-\left(\alpha_{w}+1\right)}-r_{t} x^{n_{t}-\left(\alpha_{w}+1\right)} \in R y$. If $n_{t}>\alpha_{w}+1$, then $r_{t} \in R y$, which is a contradiction; and if $n_{t}=\alpha_{w}+1$, then $1 \in M$, again a contradiction.

CASE 2. $n_{t}<\alpha_{w}+1$. Multiplication of (3) by $y^{\alpha_{w}}$ shows that $x^{\alpha_{w}+1} \in R y$, a contradiction.

Hence, $I$ is not finitely generated, and so $S$ is not coherent, a contradiction to our original assumption.

REMARK 1. The proof of Theorem 1 provides a constructive means of finding a non-coherent overring $S$ for a Noetherian domain of dimension greater than or equal to 2 . A bit more can be said about such an $S$ after one recalls the following definition. A domain $R$ is said to be finite-conductor (2-coherent) if 
( $a: b$ ) is a finitely generated ideal of $R$ for each $a, b \in R$ (equivalently, if each $R$

2-generated ideal of $R$ is finitely presented). Finite-conductor domains have figured recently in [6] and [3]. It is apparent that any coherent domain is finite-conductor, while it is well known that the converse is not generally true. By examining the proof of Theorem 1 , it is evident that the above mentioned $S$ is not finite-conductor.

REMARK 2. A natural question to consider is the following:

(a) If each overring of $R$ is coherent, is $R$ treed? ( $R$ is said to be treed if $\operatorname{Spec}(R)$, as a poset under $\subseteq$, is a tree.)

One could not expect the dimension of such an $R$ to be at most 1 , since any Prüfer domain satisfies the required hypothesis. Examples given by Dobbs and this author [3] provide a class of (non-Noetherian, non-Prüfer) treed domains satisfying the hypothesis of (a). An affirmative answer to question (a) would generalize Theorem 1.

Finally we raise the question of whether the converse of (a) is true. More precisely: if $R$ is a treed coherent domain, is each overring of $R$ coherent? With the additional assumption of $R$ being integrally closed, the converse of (a) is true, and follows directly from [6, Theorem 1].

\section{REFERENCES}

1. S. U. Chase, Direct products of modules, Trans. Amer. Math. Soc., 97 (1960), 457-473.

2. E. D. Davis, Overrings of commutative rings I, Noetherian overrings, Trans. Amer. Math. Soc., 104 (1962), 52-61.

3. D. E. Dobbs and I. J. Papick, When is $D+M$ coherent? Proc. Amer. Math. Soc., 56 (1976), 51-54.

4. R. Gilmer, Multiplicative ideal theory. Queen's Papers in Pure and Appl. Math., No. 12, Queen's University, Kingston, Ontario, 1968.

5. I. Kaplansky, Commutative Rings, Allyn and Bacon, Boston, Massachusetts, 1970.

6. S. McAdam, Two conductor theorems, J. Algebra, 23 (1972), 239-240.

7. M. Nagata, Local Rings, Interscience Publishers, New York, 1962.

ADELPHI UNIVERSITY

GARDEN CITY,

NEW YORK 11530 